\title{
O papel dos estados afetivos sobre os processos cognitivos de assimilação e acomodaçãa ${ }^{1}$
}

\author{
Maura Ribeiro Alves \\ Renata Ferrarez Fernandes Lopes ${ }^{2}$ \\ Ederaldo José Lopes \\ Universidade Federal de Uberlândia, Uberlândia-MG, Brasil
}

\begin{abstract}
Resumo: Este artigo objetivou investigar se o humor positivo induz ativação de conhecimento prévio (função assimilativa) e se o humor negativo aciona processos cognitivos dirigidos pelos dados (função acomodativa). A amostra foi composta de 32 participantes, de ambos os sexos, com idade entre 17 e 38 anos. O experimento testou o efeito de geração ativa de conhecimento. Induziram-se inicialmente humores positivos e negativos nos participantes. A tarefa final consistiu em recordar livremente uma lista de palavras completas (favorecendo processos dirigidos pelos dados) ou incompletas (favorecendo processos cognitivos dirigidos por conhecimento prévio via geração ativa), apresentadas anteriormente. Sob humor positivo, os participantes recordaram mais palavras completadas por eles durante a fase de codificação (efeito de geração ativa), sugerindo prevalência de funções assimilativas. Sob estado de humor negativo, recordaram mais palavras completas oferecidas pelo experimentador, indicando predomínio de funções acomodativas. Conclui-se que os estados afetivos atuam sobre a seleção e regulação dos processos cognitivos de assimilação e acomodação.
\end{abstract}

Palavras-chave: afeto, humor, processos cognitivos.

\section{The role of affective states on cognitive processes of assimilation and accommodation}

\begin{abstract}
This study investigated whether a positive mood induces the activation of previously acquired knowledge (assimilative function) and whether a negative mood triggers data-driven cognitive processing (accommodative function). The sample was composed of 32 female and male individuals, between 17 and 38 years of age. The experiment tested active generation effect of knowledge. Initially, positive and negative moods were induced in the participants. The final task consisted of freely recalling a previously presented list of complete words (favoring data-driven processing) or incomplete ones (favoring cognitive processes driven by previous knowledge through active generation). The participants recalled more words completed by them during the coding stage (active generation effect) under a positive mood, suggesting the prevalence of assimilative functions. Under a negative mood however, they recalled more complete words provided by the researcher, indicating a predominance of accommodative functions. The study's results suggest that affective states act upon the selection and regulation of cognitive processes of assimilation and accommodation.
\end{abstract}

Keywords: affection, mood, cognitive processes.

\section{El papel de los estados afectivos en los procesos cognitivos de asimilación y acomodación}

Resumen: En este trabajo fue investigado si el humor positivo induce la activación de conocimiento previo (función asimilativa) y si el humor negativo acciona procesos cognitivos dirigidos por los datos (función de acomodación). Participaron en esta investigación 32 voluntarios entre 17 y 38 años de edad, hombres y mujeres. La experimentación probó el efecto de la generación activa del conocimiento. Fueron inducidos inicialmente humores positivos y negativos en los participantes. La tarea final consistió en recordarse libremente de una lista de palabras completas (favoreciendo procesos orientados por los datos) o incompletas (favoreciendo procesos cognitivos orientados por conocimientos previos a través de la generación activa) presentadas anteriormente. Bajo el humor positivo, los participantes recordaron de más palabras completadas por ellos a lo largo de la fase de codificación (efecto de la generación activa), lo que sugiere la predominancia de las funciones asimilativas. Bajo el estado de humor negativo, recordaron de más palabras completas ofrecidas por el experimentador, lo que indica el predominio de las "funciones de acomodación". Se concluye que los estados afectivos actúan en la selección y regulación de los procesos cognitivos de asimilación y acomodación.

Palabras clave: afección, humor, procesos cognitivos.

\footnotetext{
${ }^{1}$ Este texto foi revisado seguindo o Acordo Ortográfico da Língua Portuguesa (1990), em vigor a partir de $1^{\circ}$ de janeiro de 2009. Artigo derivado da dissertação de mestrado da primeira autora sob orientação da segunda, com financiamento da CAPES. Agradecimentos são devidos ao CNPq e à FAPEMIG pelo apoio financeiro à segunda autora.

${ }^{2}$ Endereço para correspondência:

Renata Ferrarez Fernandes Lopes. Universidade Federal de Uberlândia. Instituto de Psicologia. Avenida Pará 1720/Bloco 2C. CEP 38.405-320. Uberlândia-MG, Brasil.E-mail: rfernandeslopes@fapsi.ufu.br
}

A relação entre cognição e afeto foi negligenciada nos primórdios do movimento da psicologia cognitiva moderna ou processamento de informação (Gardner, 2003), mas adquiriu importância nas últimas três décadas (Forgas, 2008a, 2008b; Frijda, Manstead, \& Bem, 2000). Um dos aspectos observados nos estudos que investigam tal relação diz respeito ao papel do humor sobre os processos cognitivos, 
especificamente em relação aos processos de assimilação e acomodação (Bless \& Fiedler, 2006; Fiedler \& Bless, 2000; Forgas, 2007; Lopes \& Alves, 2009; Pretz, Totz, \& Kaufman, 2010). Fiedler e Bless (2000; ver também Ashton-James \& Ashkanasy, 2008; Bäuml \& Kuhbandner, 2010; Pretz e cols., 2010) mostraram que o estado de humor pode influenciar performances cognitivas, estilos de processamento, processos de memória, bem como tem papel fundamental na seleção e na regulação adaptativa dos processos cognitivos.

Em qualquer processo cognitivo, há dois componentes que correspondem à acomodação e assimilação: a conservação e a geração ativa. O processo conservativo significa codificar as informações vindas dos estímulos externos e recuperar informações da memória interna (ou seja, usar a memória de trabalho). Assim, aperfeiçoar o desempenho conservativo é aumentar a fidelidade ao estímulo, ficar preso aos fatos e minimizar a perda da informação, o que reflete pura acomodação. Por outro lado, a geração ativa é governada pelas influências top-down de estruturas de conhecimento pré-existentes. Esse componente expressa funções assimilativas e gerativas como transformação da informação, construção de inferências, pensamentos produtivos ou criatividade (Fiedler \& Bless, 2000). O modelo teórico de Fiedler e Bless (2000) fundamentou-se nos estudos de Piaget (1954), acerca dos dois processos de regulação adaptativa: assimilação e acomodação. A assimilação ocorre quando o organismo assimila estímulos externos, a partir de sua estrutura interna, o que pode ser descrito como um processamento top-down (dirigido pelo conhecimento prévio) no qual o indivíduo impõe suas estruturas e esquemas cognitivos que foram empregados com sucesso no passado para resolver novos problemas, refletindo um processo adaptativo de autoconfiança, baseado nos valores e estratégias comportamentais previamente aprendidos. Por outro lado, na acomodação, o organismo é dirigido ou governado pelas propriedades dos estímulos (processamento bottom up). Esse tipo de processamento de informação caracteriza-se pela prontidão do organismo em reagir seguramente a demandas ou ameaças externas. Assim, o processo de acomodação implica prender-se aos estímulos e atualizar as estruturas internas como uma função das exigências externas. Os dois processos cognitivos têm funções diferentes, mas são indissociáveis (Fiedler, Bluemke, \& Unkelbach, 2009). Piaget (1952) afirma que a assimilação ou a acomodação pode ter maior dominância em função das demandas do ambiente.

Outra sustentação teórica do modelo de Fiedler e Bless (2000) refere-se à visão comportamentalista no que tange a relacionar o estado de humor a comportamentos específicos. Comportamentalistas afirmam que aprendizagens prazerosas (sob humor positivo) são caracterizadas por comportamentos de exploração, curiosidade, autoeficiência e outros que requerem assimilação, enquanto comportamentos aversivos são dirigidos por atenção cautelosa e por aprendizagens de evitação, sendo estes exemplos de funções acomodativas (Higgins, Roney, Crowe, \& Hymes, 1994).
Baseando-se nessas concepções, Fiedler e Bless (2000) propuseram um modelo teórico afirmando que os estados afetivos positivos parecem ativar processos de assimilação. Assim, quando um indivíduo está em um estado de afeto positivo, ele confia mais em seu conhecimento internalizado. Dado que o processamento top-down serve como uma função econômica sobre os recursos mnemônicos, o indivíduo tem maiores condições de explorar curiosamente o ambiente e resolve problemas de forma mais criativa (Bless \& Fiedler, 2006). Por outro lado, os estados afetivos negativos parecem ativar os processos acomodativos. Esses processos poderiam ser inferidos a partir dos comportamentos observados sob um estado de humor ou outro. Considerando-se que uma pessoa que se encontra em um estado de afeto negativo está presa à informação do ambiente, ela se torna mais cautelosa, cuidadosa e receosa, prejudicando as habilidades mais eficazes de resolução de problemas (Fiedler \& Bless, 2000; Wyland \& Forgas, 2010). Bless e cols. (1996) induziram humores tristes ou alegres em dois grupos de participantes e apresentaram a eles, por meio de uma gravação, informações sobre scripts de atividades bem conhecidas (como, “jantar"). Alguns dos scripts eram típicos (por exemplo, sentar-se à mesa, pegar o prato, colocar a comida) e outros eram atípicos (como, colocar guardanapo no colo). Após a apresentação da gravação, foi aplicado um teste surpresa de memória aos participantes acerca das atividades apresentadas. Diante dos resultados obtidos, verificou-se que os participantes com humor alegre lembraram-se mais das atividades típicas do que aqueles com humor negativo, bem como de recordações "intrusas" de atividades típicas que não foram apresentadas. Esses resultados mostram que o estado de humor positivo aciona, com maior prevalência, as estruturas antigas de conhecimento internalizado (crenças) e enfatizam a confiança que os participantes com humor positivo têm em relação a essas estruturas, em comparação aos participantes em um estado de humor negativo.

Bless, Schwarz e Wieland (1996) avaliaram o impacto dos estados de humor sobre as funções assimilativas, testando o efeito do estereótipo (crenças sobre grupos sociais). Eles pressupunham que, em um julgamento sobre um dado estereótipo, os indivíduos sob estado de humor positivo seriam guiados por crenças vinculadas a esse estereótipo e, caso surgisse uma informação inconsistente a ele, esta seria ignorada. Para verificar essas questões teóricas, os pesquisadores elaboraram grupos de personagens com estereótipos bem definidos (como: ladrão, político, traficante) listando uma série de informações sobre esses. Alguns personagens foram apresentados com as informações consistentes ao estereótipo (como, por exemplo, ladrão: morador de periferia, negro, mulato, pobre, semianalfabeto), outros apareceram associados a informações inconsistentes (por exemplo: ladrão: morador de periferia, negro, mulato, curso superior). Os resultados alcançados, em relação à condição de informação consistente ao estereótipo, mostraram que os participantes com humor positivo recordam-se mais de julgamentos consistentes do que aqueles com humor 
negativo. Já os resultados para informação inconsistente mostraram que os indivíduos com humor positivo utilizaram um processamento maior para a informação discrepante. Esses dados demonstraram a ativação de estruturas de conhecimento internas, com maior prevalência sob humor positivo, para que os participantes tomassem uma decisão que implicaria num julgamento consistente ao estereótipo, indicando a relação que pode existir entre os estados de humor positivo e as funções assimilativas.

Em outro experimento realizado por Fiedler, Nickel, Asbeck e Pagel (2003), apresentou-se aos participantes uma lista de verbos precedidos de substantivos semanticamente equivalentes. Os participantes tinham de ler os pares associados (substantivos-verbos), sendo que metade desses verbos tinha de ser gerada a partir das letras iniciais. Antes da apresentação das palavras, foram induzidos estados afetivos positivos e negativos em igual número de participantes. No final do experimento, foi feito um teste de recordação. O resultado indicou que os participantes sob estado positivo recordaram-se mais das palavras geradas, ao passo que aqueles com humor negativo recordaram-se mais das palavras lidas.

Este artigo apresenta um experimento cujo objetivo foi investigar a relação do estado de humor com os processos cognitivos assimilativos e acomodativos, por meio de tarefa de geração ativa. Mais especificamente, o experimento testou se o conhecimento geral internalizado é facilitado por processos assimilativos e é ativado quando o indivíduo está em um estado de humor positivo e se o conhecimento dirigido pelas demandas externas (facilitado por funções acomodativas) é suscitado por um estado de humor negativo.

\section{Método}

\section{Participantes}

Este experimento foi realizado em 32 participantes com idade entre 17 e 38 anos $(M=27,5 ; D P=14,84)$, de ambos os sexos (4 homens e 28 mulheres), que cursam ou cursaram o ensino superior. Desse total, 22 participantes (3 homens e 19 mulheres), com idade média entre 17 e 38 anos $(M=27,5$ anos; $D P=14,85)$, realizaram o experimento com a tarefa distraidora, enquanto 10 participantes ( 1 homem e 9 mulheres), com idade média entre 18 e 30 anos ( $M=24$ anos; $D P=$ 8,48 ), realizaram o experimento sem a tarefa distraidora. Em cada um dos grupos, metade dos participantes passou pelo processo de indução de humor negativo e metade pelo processo de indução de humor positivo. Trata-se de uma amostra de conveniência, e a diferença numérica dos dois grupos de participantes ocorreu porque foi necessário excluir oito participantes da análise dos dados no grupo experimental por se tratar de casos que apresentaram escores atípicos na escala de indução de humor.

\section{Materiais}

Para a realização do experimento, utilizou-se uma lista de 96 palavras composta por substantivos (48) e verbos (48), metade deles com valência positiva e metade com valência negativa. A valência das duas categorias de palavras foi baseada no estudo de Rossel e Nobre (2004). Os substantivos eram semanticamente semelhantes ao verbo correspondente e tinham como finalidade a função de priming. Essa lista foi obtida a partir de uma prova de juízes psicólogos (peritos), cujo critério de concordância acerca da semelhança semântica entre as palavras foi superior a $60 \%$. Esse procedimento foi baseado em Pinho e Guzzo (2003). Entretanto, o critério de concordância entre os juízes, adotado pelas autoras, foi $50 \%$. Dos 48 verbos, metade estava com a grafia completa (apresentando todas as letras), e a outra metade estava com a grafia incompleta (faltando algumas letras). Para exemplificar, o verbo estragar foi apresentado com a grafia completa (estragar), já o verbo amar foi apresentado com a grafia incompleta, como, por exemplo, $\left(a \_m \_r\right)$. As palavras também foram divididas em valências positivas e negativas, havendo, portanto, 24 pares de substantivos-verbos com conteúdo positivo (por exemplo: afeição - amar; auxílio - ajudar; canção - cantar; confiança - confiar; proteção - defender etc.) e 24 substantivos-verbos com conteúdo negativo (por exemplo: ataque - agredir; assassinato - matar; intimidação - ameaçar; punição - castigar; menosprezo - desprezar; aversão - detestar etc.), totalizando 96 palavras.

Para a avaliação do humor, utilizou-se uma escala Likert de 5 pontos em que o participante escolhia uma das alternativas que melhor descrevia seu estado de humor naquele exato momento. As alternativas foram as seguintes: muito triste (1), triste (2), nem triste e nem alegre (3), alegre (4), muito alegre (5). A tarefa de distração consistiu na apresentação de um filme por meio do microcomputador com duração de aproximadamente 10 minutos, intitulado "Marte: o planeta vermelho". Esse filme é um documentário sobre como o planeta Marte se formou, suas características geológicas, físico-químicas e biológicas, bem como suas similaridades e diferenças com o planeta Terra. O objetivo dessa tarefa foi restringir o efeito da indução do humor à fase de codificação e armazenamento, neutralizando o humor antes de dar início à fase de recuperação da informação. As instruções e os estímulos foram apresentados por meio do programa Superlab-Pro $\$ (Cedrus Corporation, 2002), em um computador devidamente configurado para sua execução.

\section{Procedimento}

\section{Coleta de dados}

O primeiro procedimento aplicado foi a tarefa de indução do humor que consistiu em pedir para o participante escrever em uma folha de papel em branco sobre uma experiência triste ou feliz que ocorreu em sua vida, nos últimos três anos. Essa etapa teve como finalidade induzir o humor na mesma valência do conteúdo da história redigida. Assim a indução do humor positivo deu-se pela recordação e redação da experiência feliz nos últimos três anos, ao passo que o humor negativo foi induzido pela recordação e redação da 
experiência triste ocorrida nesse mesmo período. O procedimento para indução do humor foi elaborado pelos autores com base no modelo de infusão de afeto de Forgas (2000). Segundo ele, várias experiências cotidianas (por exemplo: assistir a um filme, caminhar em um dia ensolarado, ouvir uma música e se lembrar de experiências episódicas) são capazes de produzir ou mudar um estado de humor. Dessa forma, a redação de um episódio de intensa experiência afetiva é uma tarefa potencialmente capaz de induzir diferentes estados de humor. Finalmente, aplicou-se a escala de avaliação do humor. Esses dois procedimentos foram aplicados a todos os participantes.

Após a etapa de avaliação do humor dos participantes, realizou-se a aplicação do experimento de geração ativa de forma individual. Iniciou-se a apresentação dos estímulos com um treino contendo seis provas. Cada verbo (completo ou incompleto) foi precedido por um substantivo relacionado a ele semanticamente e de mesma valência. A ordem de apresentação foi substantivo-verbo até o término do experimento. A tarefa consistia em ler o substantivo e, em seguida, ler ou gerar um verbo, sempre em voz alta. Por exemplo, nas provas envolvendo geração ativa, o participante deveria, por exemplo, ler o substantivo (canção) em voz alta e, em seguida, gerar o verbo cantar (c_n_t__ $)$. As letras que compunham os verbos incompletos eram intercaladas com os travessões. Nas provas em que não se exigiu a geração ativa, a tarefa consistia em ler um substantivo e, na sequência, um verbo (ataque - agredir). $\mathrm{O}$ tempo de apresentação de cada palavra (substantivo e verbo), na tela do computador, foi de 6 segundos, tanto para os 24 verbos completos como para os 24 incompletos. Os estímulos foram apresentados de forma aleatória, ou seja, para cada participante foi apresentada uma ordem diferente da sequência dos pares substantivos-verbos.

$\mathrm{Na}$ condição em que se utilizou a tarefa distraidora, o participante assistiu a um trecho do filme intitulado "Marte: o planeta vermelho", com duração de 10 minutos e, em seguida, respondeu a cinco questões de múltipla escolha relacionadas ao filme, a saber: "O nome do planeta Marte é originado do..."; "Em Marte está a maior montanha do sistema solar, qual o seu nome?”; "Quais as condições climáticas de Marte?"; "Qual o tamanho do planeta Marte?”; e "O planeta Marte está desprovido de...”. Para todas essas perguntas, o participante devia assinalar uma resposta entre quatro alternativas. O objetivo dessa tarefa era estabilizar o humor antes do início da fase de recuperação dos dados, fazendo com que o participante permanecesse em um estado de humor neutro durante a fase de recuperação da informação que consistia numa tarefa de recordação livre. Dessa forma, os efeitos da indução do humor ficariam restritos às etapas de codificação e armazenamento dos dados. Em seguida, realizou-se novamente a avaliação do estado afetivo do participante por meio da mesma escala de humor utilizada previamente.

Na condição experimental na qual não se utilizou a tarefa distraidora, os participantes realizaram a tarefa de geração, porém não assistiram ao trecho do filme sobre Marte. Nessa condição substituiu-se o filme pela leitura da história escrita pelo participante na fase de indução do humor, reforçando o estado de humor (tanto positivo quanto negativo) originalmente induzido. A leitura foi seguida por uma nova avaliação do humor, antes do teste de recordação livre.

Para finalizar o experimento, todos os participantes foram submetidos a um teste de recordação livre. A experimentadora deu-lhes uma folha em branco e pediu para escreverem, em qualquer ordem, todos os verbos apresentados, ou seja, grafados de forma completa (lidos) ou incompleta (gerados) de que eles se lembrassem.

\section{Análise dos dados}

A percentagem de recordações corretas de verbos lidos ou gerados foi analisada com estatística não paramétrica, uma vez que a análise de assimetria (skewness) indicou valores de $\chi^{2}$ inferiores a 0,24 (Miles \& Shevlin, 2001). Considerandose tal assimetria na distribuição dos dados, empregaram-se o teste de Mann-Whitney, para a comparação de amostras não relacionadas, e o teste de Wilcoxon, quando as amostras eram relacionadas.

\section{Considerações éticas}

O projeto de pesquisa que originou este artigo foi aprovado pelo Comitê de Ética em Pesquisa da Universidade Federal de Uberlândia (Processo CEP/UFU no 405/08). Todos os participantes da amostra assinaram o Termo de Consentimento Livre e Esclarecido (TCLE), antes de iniciar a pesquisa.

\section{Resultados}

\section{Análise da efetividade da indução de humor}

Uma análise da condição de humor positivo, com tarefa distraidora presente (filme antes da tarefa de livre recordação), mostrou que não houve diferença estatística entre as duas medidas de humor realizadas $(z=1,66 ; p=0,096)$, sendo que a primeira medida, realizada logo após a indução do humor, apresentou mediana de 4,09, e a segunda medida, realizada logo após a tarefa distraidora, apresentou mediana de 3,63. A análise da condição de humor negativo, com tarefa distraidora presente, mostrou que houve diferença estatística entre as duas medidas realizadas $(z=$ 2,$8 ; p=0,05$ ). Os dados indicaram que houve melhora do humor de quase 1 ponto, da primeira (mediana $=3,18)$ para a segunda medida (mediana $=4,09$ ).

$\mathrm{Na}$ condição experimental em que a indução do humor foi positiva, mas não se utilizou a tarefa distraidora (e pediuse para o participante reler sua redação sobre o evento prazeroso que the acontecera imediatamente antes do teste de recordação livre), os resultados mostraram que não houve 
diferença estatística $(z=0,57, n s)$ entre as duas medidas realizadas (primeira medida: mediana $=4,2$ e segunda medida: mediana $=4,0)$. Quando as mesmas análises anteriores foram feitas com os dados obtidos sob estado de humor negativo, sem tarefa distraidora (releitura da história triste), observou-se que o estado de humor negativo piorou da primeira avaliação (mediana $=3,4)$ para a segunda avaliação $($ mediana $=2,6)$.

\section{Análise do desempenho na tarefa de recordação livre dos verbos lidos}

Não houve diferença na recordação dos verbos lidos em um estado de humor positivo (mediana $=5,1$ ) comparativamente a um estado de humor negativo (mediana $=6,6$ ), com $z=0,89$, $n s$, na condição em que a tarefa distraidora estava presente. Na ausência da tarefa distraidora, os resultados mostraram que a recordação dos verbos lidos é maior no estado de indução de humor negativo, $\operatorname{com} z=2,41, p=$ 0,0016. O índice mediano de recordação de verbos lidos sob estado de humor negativo foi de 9,2 e para os verbos lidos sob estado de humor positivo foi de 4,0 .

\section{Análise do desempenho na tarefa de recordação livre dos verbos construídos}

Na presença da tarefa distraidora, os resultados mostraram que houve diferença estatística entre as duas condições de humor testadas. Houve maior eficiência na recordação das palavras construídas, sob humor positivo (mediana $=7,09$ ), comparativamente à recordação de palavras construídas, sob estado de humor negativo (mediana $=5,9$ ), $\operatorname{com} z=0,95$; $p=0,038$. Na ausência da tarefa distraidora, os resultados mostraram que também houve diferença estatística entre as duas condições de humor testadas, $\operatorname{com} z=1,16, p=0,024$, indicando vantagem para a recordação de verbos construídos sob estado de humor positivo (mediana $=9,8$ ) em relação àqueles construídos sob humor negativo (mediana $=6,6)$.

\section{Análise do desempenho na tarefa de recordação livre dos verbos lidos versus construídos}

Na presença da tarefa distraidora, os resultados mostraram que não houve diferença estatística entre as duas tarefas testadas (recordação livre de verbos lidos: mediana $=5,1$, e a recordação de verbos construídos: mediana $=7,09)$, $\operatorname{com} z=$ $0,71, n s$, sob humor positivo. Da mesma forma, nessa condição experimental, não houve diferença estatística significante $(z=0,29, n s)$ para a recordação de verbos lidos (mediana $=6,6)$ comparativamente à recordação de verbos construídos (mediana $=5,9)$, sob humor negativo. Na ausência da tarefa distraidora, os resultados mostraram que houve diferença estatística significante entre as duas condições testadas: a recordação livre de verbos lidos foi inferior (mediana $=4,0$ ) à recordação dos verbos construídos (mediana $=9,8)$, sob o humor positivo, $\operatorname{com} z=0,37 ; p=0,050$. A diferença estatística também ocorreu entre a recordação livre de verbos lidos $($ mediana $=9,2)$ em relação aos verbos construídos (mediana $=6,6$ ), sob o humor negativo, $\operatorname{com} z=1,60 ; p=0,010$.

\section{Discussão}

Os dados mostram que, em geral, a tarefa distraidora age efetivamente sob estados de humor negativo. Na condição de humor positivo, ela não parece ter alterado tal estado, mas, com relação ao humor negativo, nota-se que houve alteração desse estado de humor da primeira para a segunda medida, ou seja, a tarefa distraidora parece ter neutralizado o estado de humor, que de triste passou para neutro. Todavia, na ausência da tarefa distraidora, o humor negativo piora da primeira para a segunda avaliação. Esses dados vão ao encontro da literatura sobre os efeitos do estado de humor sobre o processamento de informação (Bless \& Fiedler, 2006, Fiedler $\&$ Bless, 2000). Isto é, estados de humor positivo levam a processos assimilativos, o que explica a ausência de efeitos da tarefa distraidora nesses estados. Sob humor positivo, os participantes processam a informação externa sem esforço ou demandas atencionais. Se a informação externa não captura a atenção (processo automático), o processamento da informação pode basear-se num processo heurístico e intuitivo, portanto sujeito a processos esquemáticos, a exemplo do que ocorre em tarefas de aprendizagem implícita (Pretz e cols., 2010; Wyland \& Forgas, 2010 ).

Considerando a tarefa de recordação livre de verbos lidos, na presença da tarefa distraidora, não houve diferença entre estados de humor positivo e negativo. No entanto, na ausência da tarefa distraidora, houve uma vantagem na recordação sob o estado de humor negativo, sugerindo que o processamento nesse caso está sob o controle de variáveis externas ou processos acomodativos (Bless \& Fiedler, 2006). Verificou-se, então, que os participantes sob humor induzido negativamente apresentaram uma vantagem mnemônica com relação às palavras lidas. Considerando a prevalência de processos acomodativos, associados a um estado de humor negativo, a recordação de palavras lidas (tarefa predominantemente acomodativa) sob humor negativo permite inferir que houve um direcionamento bottom up do processamento da informação, ou seja, os participantes foram "dirigidos pelos dados" (Fiedler e cols., 2003). Para Isen e Daubman (conforme citado por Isen, 1987), as pessoas sob estado de humor negativo podem estar mais motivadas a reparar seu humor, tentando parar os processos de pensamento que podem suscitar a tristeza. Os dados apresentados neste trabalho mostram que a presença da tarefa distraidora parece neutralizar o estado de humor negativo induzido, ao mesmo tempo em que a ausência da tarefa distraidora facilita a leitura, mas não a geração (construção) dos verbos, sob esse mesmo estado de humor. 
Com relação à tarefa de geração ativa, observa-se a vantagem na recordação dos verbos construídos sob humor positivo, tanto na ausência quanto na presença da tarefa distraidora. Assim, verificou-se que, num estado de humor positivo, uma tarefa que demanda funções assimilativas de criação e construção possivelmente proporcionou um direcionamento do processamento da informação para funções de prevalência top-down, ou seja, os participantes, nesse contexto de humor positivo, reportaram-se mais ao conhecimento prévio acerca do estímulo apresentado (par de substantivo e verbo), ativando um maior número de informações ligadas a esses estímulos (redes semânticas que facilitariam a recordação dos verbos construídos) (Bower, 1981). Como indicação dessa ativação em rede melhorando a capacidade de recordação, o resultado do teste de recordação livre para palavras construídas mostra que, sob humor positivo (sem o uso da tarefa distraidora), os participantes lembraram-se mais das palavras construídas, enquanto as palavras lidas foram recordadas de forma mais eficiente, sob humor negativo. Alguns estudos confirmaram o mesmo tipo de processamento observado nesta pesquisa: a ativação de conhecimento prévio (crenças) e a vantagem sobre funções assimilativas estão mais associadas ao estado de humor positivo do que ao humor negativo (Bless \& Fiedler, 1995; Fiedler e cols., 2003).

Por exemplo, Bless e Fiedler (1995) realizaram uma pesquisa utilizando a tarefa de verificação de sentença que teve como finalidade capturar o julgamento ativado por estruturas de conhecimento previamente eliciadas (esquemas/ crenças). Para eles essas estruturas têm duas características importantes: (1) elas permitem reduzir a complexidade do processamento da informação, ou seja, no estágio de codificação o esquema permite ignorar detalhes insignificantes das situações, influenciando o processo de recuperação; (2) permitem fazer inferências além das informações dadas, sendo assim, a estrutura de conhecimento geral ativa o processo inferencial (ativação em rede). Segundo os autores, a formação de um julgamento anterior pode facilitar o julgamento subsequente através de dois processos: transferência (resultado da repetição, ou seja, o resultado do primeiro julgamento é conservado e transferido ao segundo julgamento, porém esse processo requer congruência de julgamento) e inferências de geração, processo pelo qual o indivíduo pode formar o segundo julgamento mais rapidamente devido a inferências provocadas pela ativação de estruturas de conhecimento ativadas durante o julgamento anterior. Nesse caso, o efeito de facilitação (tempo menor de latência para o segundo julgamento) é devido à base informacional e não à memorização do resultado do primeiro julgamento e deve ser observado em julgamentos incongruentes.

\section{Considerações finais}

Os resultados apresentados mostram que processos assimilativos operam sob estados de humor positivo, enquanto processos acomodativos operam sob estados de humor negativo. Todavia, é preciso investigar outras variáveis que estão além dos processos assimilativos e acomodativos e que podem exercer influência na relação afeto-cognição, como, por exemplo, o efeito de neutralização do humor negativo e suas consequências na tarefa de recordação livre de palavras lidas ou geradas. Esse efeito pode ser observado pelo emprego da tarefa distraidora e merece investigações futuras, pois parece exercer influência sobre a congruência do humor (Fiedler e cols., 2003).

Finalmente cabe ressaltar as consequências deste tipo de pesquisa e dos resultados obtidos. Processos assimilativos e acomodativos são igualmente importantes na vida cotidiana. Um processo não é mais importante que o outro. Ambos operam no sentido da adaptação e da homeostase. Seja no mundo do trabalho, do ensino-aprendizagem e de contextos clínicos, muitos problemas podem ser resolvidos estimulando-se a criatividade e o uso de novas heurísticas contextualizadas às demandas, mas em certas situações o cuidado com os riscos apresentados e as possibilidades de insucessos frente às demandas podem exigir que a pessoa seja mais cuidadosa na análise do problema e na tomada de decisão. Em ambas as situações, os processos assimilativos e acomodativos podem operar igualmente bem.

\section{Referências}

Ashton-James, C. E., \& Ashkanasy, N. M. (2008). Affective events theory: A strategic perspective. In W. J. Zerbe, C. E. J. Hartel, \& N. M. Ashkanasy (Eds.), Research on emotion on organization. Emotions, ethics, and decision making (pp. 1-34). Bingley, UK: Emerald Group Publishing/JAI Press.

Bäuml, K. H., \& Kuhbandner, C. (2007). Remembering can cause forgetting, but not in negative moods. Psychological Science, 18(2), 111-115.

Bless, H., \& Fiedler, K. (1995). Affective states and the influence of activated general knowledge. Personality and Social Psychology Bulletin, 21(7), 766-778.

Bless, H., \& Fiedler, K. (2006). Mood and the regulation of information processing and behavior. In J. P. Forgas (Ed.), Affect in social cognition and behaviour (pp. 6584). New York: Psychology Press.

Bless, H., Clore, G. L., Schwarz, N., Golisano, V., Rabe, C., \& Wölk, M. (1996). Mood and the use of scripts: Does happy mood make people really mindless? Journal of Personality and Social Psychology, 71(4), 585-595.

Bless, H., Schwarz, N., \& Wieland, R. (1996). Mood and the impact of category membership and individuating information. European Journal of Social Psychology, 26(6), 935-959.

Bower, G. H. (1981). Mood and memory. American Psychologist, 36(2), 129-148.

Cedrus Corporation. (2002). SuperLab: Experimental Laboratory Software. San Pedro: Cedrus. 
Fiedler, K., \& Bless, H. (2000). The formation of beliefs at the interface of affective and cognitive processes. In N. H. Frijda, A. S. R. Manstead, \& S. Bem (Eds.), Emotions and beliefs: How feelings influence thoughts (pp. 144170). New York: Cambridge University Press.

Fiedler, K., Nickel, S., Asbeck, J., \& Pagel, U. (2003). Mood and the generation effect. Cognition and Emotion, 17(4), 585-608.

Fiedler, K., Bluemke, M., \& Unkelbach, C. (2009, março). Exerting control over allegedly automatic associative processes. Trabalho apresentado no Symposium on Social Psychology, Sydney, Australia.

Forgas, J. P. (2000). Feeling is believing? The role of processing strategies in mediating affective influences on beliefs. In N. H. Frijda, A. S. R. Manstead, \& S. Bem (Eds.), Emotions and beliefs: How feelings influence thoughts (pp. 108-143). New York: Cambridge University Press.

Forgas, J. P. (2007). When sad is better than happy: Negative affect can improve the quality and effectiveness of persuasive messages and social influence strategies. Journal of Experimental Social Psychology, 43(4), 513-528.

Forgas, J. P. (2008a). Affect and cognition. Perspectives on Psychological Science, 3(2), 94-101.

Forgas, J. P. (2008b). Research on affect and social behavior: Links to cognitive, learning, and neuropsychology. In P. A. M. Van Lange (Ed.), Bridging social psychology benefits of transdisciplinary approaches (pp. 117-122). New Jersey: Taylor \& Francis.

Frijda, N. H., Manstead, A. S. R., \& Bem, S. (2000). The influence of emotions on beliefs. In N. H. Frijda, A. S. R. Manstead, \& S. Bem (Eds.), Emotions and beliefs: How feelings influence thoughts (pp. 1-9). New York: Cambridge University Press.

Gardner, H. (2003). A nova ciência da mente: Uma história da revolução cognitiva (3a ed.). São Paulo: Ed. USP.

Higgins, E. T., Roney, C. J. R., Crowe, E., \& Hymes, C. (1994). Ideal versus ought predilections for approach and avoidance: Different self-regulatory systems. Journal of Personality and Social Psychology, 66(2), 276-286.

Isen, A. M. (1987). Positive affect, cognitive processes, and social behavior. In L. Berkowitz (Ed.), Advances in experimental social psychology (pp. 203-253). San Diego: Academic Press.

Miles, J. N. V., \& Shevlin, M. E. (2001). Applying regression and correlation: A guide for students and researchers. London: Sage.

Piaget, J. (1952). The origins of intelligence in children. New York: International University Press.

Piaget, J. (1954). The construction of reality in the child. New York: Free Press.
Pinho, C. C. M., \& Guzzo, R. S. L. (2003). Taxonomia de adjetivos descritores de personalidade. Avaliação Psicológica, 2(2), 81-97.

Pretz, J. E., Totz, K. S., \& Kaufman, S. B. (2010). The effects of mood, cognitive style, and cognitive ability on implicit learning. Learning and Individual Differences, 20(3), 215-219.

Rossel, S. L., \& Nobre, A. C. (2004). Semantic priming of different affective categories. Emotion, 4(4), 354-363.

Wyland, C. L., \& Forgas, J. P. (2010). Here's looking at you kid: Mood effects on processing eye gaze a heuristic cue. Social Cognition, 28(1), 133-144.

Maura Ribeiro Alves é Mestre em Psicologia pelo Programa de Pós-graduação em Psicologia do Instituto de Psicologia da Universidade Federal de Uberlândia.

Renata Ferrarez Fernandes Lopes é Professora Associada do Instituto de Psicologia da Universidade Federal de Uberlândia.

Ederaldo José Lopes é Professor Associado do Instituto de Psicologia da Universidade Federal de Uberlândia.

Recebido: $17 / 05 / 2010$

$1^{a}$ revisão: $21 / 11 / 2010$

Aceite final: 15/02/2011 